\title{
ARBITRARY THRESHOLD WIDTHS FOR MONOTONE, SYMMETRIC PROPERTIES
}

\author{
RAPHAËL ROSSIGNOL, ${ }^{*}$ Université de Neuchâtel
}

\begin{abstract}
We show that there exist symmetric properties in the discrete $n$-cube whose threshold widths range asymptotically between $1 / \sqrt{n}$ and $1 / \log n$. These properties are built using a combination of failure sets arising in reliability theory. This combination of sets is simply called a product. Some general results on the threshold width of the product of two sets $A$ and $B$ in terms of the threshold locations and widths of $A$ and $B$ are provided.
\end{abstract}

Keywords: Threshold width; 0-1 law; parallel-series system; series-parallel system; $k$-out-of- $n$ system

2000 Mathematics Subject Classification: Primary 60F20

Secondary $60 \mathrm{E} 15 ; 60 \mathrm{~K} 10$

\section{Introduction}

Let $n$ be a positive integer, let $p$ be a real number in $[0,1]$, and denote by $\mu_{n, p}$ the probability measure on $\{0,1\}^{n}$ which is the product of $n$ Bernoulli measures with common parameter $p$. Then, for all $x=\left(x_{1}, \ldots, x_{n}\right) \in\{0,1\}^{n}$,

$$
\mu_{n, p}(x)=p^{\sum_{i=1}^{n} x_{i}}(1-p)^{\sum_{i=1}^{n}\left(1-x_{i}\right)} .
$$

We write $\mu_{p}$ instead of $\mu_{n, p}$ when no confusion is possible. For a subset $A$ of $\{0,1\}^{n}$, we say that $A$ is monotone if and only if

$$
x \in A, \quad x \preceq y \quad \Longrightarrow \quad y \in A,
$$

where ' $\preceq$ ' is the coordinate-wise partial order on $\{0,1\}^{n}$. It follows from an elementary coupling device that, for a monotone subset $A$, the mapping $p \mapsto \mu_{p}(A)$ is increasing. In many examples of interest (see Section 3), a threshold phenomenon occurs for property $A$ in the sense that the function $p \mapsto \mu_{p}(A)$ 'jumps' from near 0 to near 1 over a very short interval of values of $p$. The width of this interval is known as the threshold width (see the definition below). Such threshold phenomena have been shown to occur in most discrete probabilistic models, such as random graphs $[4$, p. 40], percolation [13, p. 52], satisfiability in random constraint models [10], [11], [5], local properties in random images [9], reliability [22], and the like. This paper deals with the problem of building properties whose threshold widths are prescribed and which, in addition, are in some sense symmetric. To make these notions precise, we first need some definitions and notation.

We say that a subset $A$ of $\{0,1\}^{n}$ is nontrivial if it is nonempty and different from $\{0,1\}^{n}$ itself. When $A$ is nontrivial and monotone, the mapping $p \mapsto \mu_{p}(A)$ is invertible, and for $\alpha \in[0,1]$ we let $p_{A}(\alpha)$ be the unique real in $[0,1]$ such that $\mu_{p(\alpha)}(A)=\alpha$. We shall sometimes

Received 5 December 2005; revision received 22 November 2006.

* Postal address: Institut de Mathématiques, Faculté des Sciences, Université de Neuchâtel, 11 rue Emile Argand, case postal 158, 2009 Neuchâtel, Switzerland. Email address: raphael.rossignol@unine.ch 


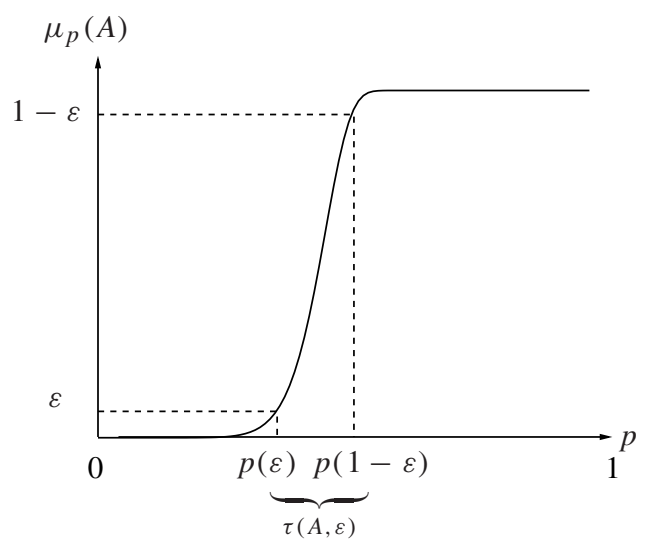

Figure 1: Example of a threshold width at level $\varepsilon$.

write $p_{A, \alpha}$ for $p_{A}(\alpha)$ and omit the subscript ' $A$ ' when there is no ambiguity. The threshold width of a subset at level $\varepsilon$ is the length of the 'transition interval', that is to say, the interval over which its $\mu_{p}$-probability increases from $\varepsilon$ to $1-\varepsilon$.

Definition 1.1. Let $A$ be a nontrivial monotone subset of $\{0,1\}^{n}$. Let $\varepsilon \in\left(0, \frac{1}{2}\right]$. The threshold width of $A$ at level $\varepsilon$ is defined by

$$
\tau(A, \varepsilon)=p(1-\varepsilon)-p(\varepsilon)
$$

(see Figure 1).

The investigation of the threshold of a monotone property, for example connectivity in a random graph, involves a sequence of nontrivial, monotone subsets $A=\left(A_{n}\right)_{n \in \mathbb{N}^{*}} \in$ $\left(\{0,1\}^{\alpha_{n}}\right)^{\mathbb{N}^{*}}$, where $\left(\alpha_{n}\right)_{n \in \mathbb{N}^{*}}$ is an increasing sequence of integers. In what follows, for technical reasons we shall suppose that $\left(\alpha_{n}\right)_{n \in \mathbb{N}^{*}}$ is only nondecreasing. Note that, in order to have an intrinsic notion of width or localisation order, we must keep in mind the respective dimensions, $\alpha_{n}$, of the spaces of which the $A_{n}$ are subsets: if the threshold width of a subset $A_{n}$ of $\{0,1\}^{\alpha_{n}}$ is of order $a(n)$, then we should properly express it as $a \circ \alpha^{-1}\left(\alpha_{n}\right)$, where $\alpha^{-1}$ is the pseudoinverse of $\alpha$, such that

$$
\alpha^{-1}(n)=\sup \left\{k \in \mathbb{N}: \alpha_{k} \leq n\right\} \quad \text { for all } n \geq \alpha_{0} .
$$

In order to describe the asymptotic behaviour of a property, we shall therefore use the following definitions.

Definition 1.2. Let $A=\left(A_{n}\right)_{n \in \mathbb{N}^{*}} \in\left(\{0,1\}^{\alpha_{n}}\right)^{\mathbb{N}^{*}}$ be a monotone property, let $a(n)$ and $b(n)$ be two sequences of real numbers in $[0,1]$, and let $\alpha \in[0,1]$. The property $A$ has a threshold located at $\alpha$ if, for all $\varepsilon \in(0,1)$,

$$
p_{A_{n}, \varepsilon} \rightarrow \alpha \quad \text { as } n \rightarrow \infty
$$

The location of $A$ is of order $a$ if, for all $\varepsilon \in(0,1)$,

$$
p_{A_{n}, \varepsilon}=O\left(a\left(\alpha_{n}\right)\right) \quad \text { as } u \rightarrow \infty .
$$


The threshold width of $A$ is of order $b$ if, for all $\varepsilon \in(0,1)$,

$$
\tau\left(A_{n}, \varepsilon\right)=O\left(b\left(\alpha_{n}\right)\right) \quad \text { as } n \rightarrow \infty .
$$

The property $A$ has a sharp threshold if, for all $\varepsilon \in(0,1)$,

$$
\frac{\tau\left(A_{n}, \varepsilon\right)}{p_{A_{n}, 1 / 2}\left(1-p_{A_{n}, 1 / 2}\right)} \rightarrow 0 \quad \text { as } n \rightarrow \infty .
$$

The property $A$ has a coarse threshold if it does not have a sharp threshold.

Intuitively it is tempting to say that a subset $A$ will have a narrow threshold unless a few coordinates have a strong influence on its definition (as an example, think of $A=\{x: x(1)=1\}$ ). In many examples, this idea is captured by the notion of symmetry.

Definition 1.3. A subset $A$ of $\{0,1\}^{n}$ is said to be symmetric if and only if there exists a subgroup $G$ of $\delta_{n}$ (the group of permutations) that acts transitively on $\{1, \ldots, n\}$ and under which $A$ is invariant, that is,

$$
g \cdot x=\left(x_{g^{-1}(1)}, \ldots, x_{g^{-1}(n)}\right) \in A \text { for all } g \in G \text { and all } x \in A .
$$

For a symmetric subset, no coordinate has a stronger influence than any other. In [12] it was proven that the threshold width of any symmetric subset $A \subset\{0,1\}^{n}$ is at most of order $1 / \log n$, and for properties whose threshold is located away from 0 and 1 it was shown that this upper bound is tight and that the threshold width is at least of order $1 / \sqrt{n}$. In order to complete these results, it is natural to ask whether, given an increasing sequence of positive real numbers $a(n)$ between $\log n$ and $\sqrt{n}$, there exists a symmetric property whose threshold width is $1 / a(n)$. This is the main question addressed in this paper.

\section{Summary and plan of the paper}

The main result of this paper is Theorem 5.1, which asserts that, under a mild hypothesis of smoothness on the sequence $a(n)$, there exists a symmetric property whose threshold width is of order $O(1 / a(n))$ when $a(n)$ lies between $\log n$ and $\sqrt{n}$. Therefore, we answer the question raised in the introduction in the affirmative. Our strategy is to construct the desired property using a suitable combination of well-known properties arising in reliability theory. Section 3 is devoted to the presentation of these properties, along with some others, which turn out to have explicitly known threshold widths and locations. The aforementioned 'suitable combination', which we shall simply call a product, will be defined, and its basic properties derived, in Section 4. Notably, it behaves particularly well with respect to the threshold widths: we shall prove that the product of two properties $A$ and $B$ has a threshold width which is the product of those of $A$ and $B$, given that the threshold of $B$ is located away from 0 and 1 . This will allow us to construct, in Section 5, a property that has a threshold width of order $O(1 / a(n))$ when $a(n)$ lies between $\log n$ and $\sqrt{n}$ (see Theorem 5.1). For the sake of completeness, in Section 6 we turn back to the theoretical study of the threshold width of the product: we examine the case in which the threshold of $B$ tends to 0 or 1 . Although we do not give a complete treatment of this case, we show that if $A$ and $B$ have thresholds respectively located at 0 and 1 , then $A \otimes B$ (their product) has a sharp threshold. 


\section{Examples of explicit threshold widths and locations}

In presenting the following examples of thresholds, our aim is twofold. First, we want to describe some of the few types of behaviour already known. Second, we shall use some of these examples in Section 5, to derive more general widths by taking products of properties.

In the framework of reliability theory, at instant $t$ two characteristic quantities of the system are especially important: the reliability, that is, the probability that no breakdown occurred before $t$, and the nonavailability, which is the probability that the system is down at instant $t$ (see, for example, [1]). Of course, these quantities differ from those in repairable systems. The analysis of the reliability of a large system, for example its asymptotic behaviour, is generally much more difficult than the analysis of the nonavailability. We shall focus only on the latter, but want to stress the fact that when dealing with a large system composed of repairable Markovian components, it is natural to expect strong similarities between the asymptotics of the two quantities (see, for example, [23]).

Let $\mathcal{A}$ denote a system composed of $n$ binary components. We can jointly describe the states of these components as a state in $\{0,1\}^{n}$, with 1 standing for a failed component and 0 standing for a working component. We can therefore associate to $\mathcal{A}$ its failure subset, which is the subset $A$ of $\{0,1\}^{n}$ containing all the configurations of the $n$ components such that the system $\mathcal{A}$ fails. If we assume that a component has failed, independently of the others, with probability $p$, then $\mu_{n, p}$ is the distribution of the state of $\mathcal{A}$ in $\{0,1\}^{n}$ and $\mu_{n, p}(A)$ is the nonavailability of $\mathcal{A}$. It is very natural to assume that the subset $A$ is monotone (if the system is down and a component fails, then the system remains down). The question of how quickly $\mu_{n, p}(A)$ 'jumps' from 0 to 1 is of great importance (see [22] for an application of [12] and [7] in this context). The main result of this article, Theorem 5.1, makes use of the properties described in Examples 3.1 and 3.2 .

Example 3.1. ( $k$-out-of- $n$ system.) The $k$-out-of- $n$ system fails when the total number of failed components is greater than or equal to a certain threshold $k \equiv k(n)$. The failure subset is therefore

$$
A_{k, n}=\left\{x \in\{0,1\}^{n}: \sum_{i=1}^{n} x_{i} \geq k\right\} .
$$

Note that the particular cases of $A_{m-1, m}$ and $A_{1, r}$ respectively correspond to parallel and series systems. Obviously, $A_{k, n}$ is monotone and invariant under every permutation of the coordinates. It is therefore a monotone, symmetric subset of $\{0,1\}^{n}$. Since the sum $\sum_{i=1}^{n} x_{i}$ has mean $n p$ and variance $n p(1-p)$ when $x$ is distributed according to $\mu_{p}$, it follows intuitively that $A_{k, n}$ has a threshold that is located at $k / n$ and has width of order $\sqrt{(k / n) \times(1-k / n)} / \sqrt{n}$. We shall make this intuition precise in Lemma 5.1, where $k=\lfloor n / 2\rfloor$. (Here $\lfloor x\rfloor$ denotes the largest integer less than or equal to $x$.)

Example 3.2. (Parallel-series system.) A parallel-series system contains $n=r \times m$ components which are assembled into $r$ blocks each containing $m$ components. The system fails as soon as a block fails, and a block fails if all of its components fail. Of course, the nonavailability of such a system is very easy to derive. Let $B_{n}$ denote its failure subset. Then

$$
\mu_{p}\left(B_{n}\right)=1-\left(1-p^{m}\right)^{r} .
$$

For example, when $m=\left\lfloor\log _{2} k\right\rfloor, r=\left\lfloor k / \log _{2} k\right\rfloor$, and $k \geq 2$, the threshold of $B_{n}$ is located at $\frac{1}{2}$ and has width of order $1 / \log n$ (see Lemma 5.2, below). Note that $B_{n}$ is monotone and symmetric (under permutation of the components inside a block and permutation of the blocks). 
Such systems, with multistate components instead of binary ones, were studied in [15] and [16], and a well-defined application was presented in [17] (see also the recent book [18]). We can also define the dual series-parallel system, in which components are assembled into $r$ blocks each containing $m$ components: the system fails when all blocks fail, and a block fails as soon as one of its components fails.

Example 3.3. (Consecutive-k-out-of- $n$ system.) In a consecutive- $k$-out-of- $n$ system the components are arranged around a circle. The system fails as soon as at least $k(n)$ consecutive components have failed. This model has asymptotic behaviour similar to that of the parallelseries system with $\lfloor n / k\rfloor$ blocks of $k$ components. For example, when $k=\left\lfloor n / \log _{2} n\right\rfloor$ the threshold of the failure subset is located at $\frac{1}{2}$ and has width of order $1 / \log n$ (for a similar result, see [22]). This model was introduced by Kontoleon [19] to model some problems arising in engineering science, such as oil transportation using pipelines, telecommunication using spacecraft relay stations, transmission of data in a ring network of computers, etc.

\section{The product of subsets of $\{0,1\}^{n}$}

To the author's knowledge, whereas the effect of simple operations between properties has been extensively studied in the context of the so-called 0-1 laws which occur in logic [8], no such work has been undertaken regarding the threshold phenomenon. The first combinations of properties that come to mind, union and intersection, behave in quite an unpleasant way with respect to the threshold width [24, Chapter 3]. In this section, we will show the nice behaviour of another combination, which we simply call the product. Even though linearity does not play any role in this setting, it is worth noting the similarity between this product and the Kronecker product of matrices. Given two properties $A$ and $B$, on two distinct spaces, their product is a property combining the elements of $A$ and $B$ in the following way.

Definition 4.1. For any subset $C$ of a set $X$, let $\mathbf{1}_{C}$ denote the characteristic function of the subset $C$ : it takes the value 1 on $C$ and the value 0 on $X \backslash C$.

Let $A$ be a subset of $\{0,1\}^{r}$ and let $B$ be a subset of $\{0,1\}^{m}$. The product of $A$ and $B$, denoted by $A \otimes B$, is the subset of $\left(\{0,1\}^{r}\right)^{m}$ defined by

$$
\eta \in A \otimes B \quad \Longleftrightarrow \quad\left(\mathbf{1}_{\left\{\eta_{1} \in A\right\}}, \ldots, \mathbf{1}_{\left\{\eta_{m} \in A\right\}}\right) \in B,
$$

where

$$
\eta=\left(\eta_{1}, \ldots, \eta_{m}\right) \quad \text { and, for all } j \in\{1, \ldots, m\}, \quad \eta_{j} \in\{0,1\}^{r} .
$$

In order to visualise the precise meaning of this definition, it is convenient to consider this product in the language of reliability theory. Let $A$ denote the failure set of a system, $\mathcal{A}$, composed of $r$ components, and let $B$ be the failure set of another system, $\mathcal{B}$, with $m$ components. Then $A \otimes B$ is the failure subset of the system obtained by replacing the components of $\mathscr{B}$ by $m$ independent copies of $\mathcal{A}$. For example, the parallel-series and seriesparallel systems can be obtained from some elementary building blocks, namely the series and parallel systems (see Figure 2). This process can be continued by embedding systems into one another (see Figure 3).

Let us now describe the basic properties of this product. A useful feature is the link between the probability of $A \otimes B$ and those of $A$ and $B$. Also, invariance and monotonicity properties for $A \otimes B$ follow if $A$ and $B$ have similar properties. Below, for $\eta=\left(\eta_{1}, \ldots, \eta_{m}\right)$ in $\left(\{0,1\}^{r}\right)^{m}$ with $\eta_{j} \in\{O, 1\}^{r}$ for every $j$, we will denote by $\eta_{i, j}$ the $i$ th coordinate of $\eta_{j}$, which is therefore 0 or 1 . In this way, we identify $\left(\{0,1\}^{r}\right)^{m}$ and $\{0,1\}^{r m}$. 


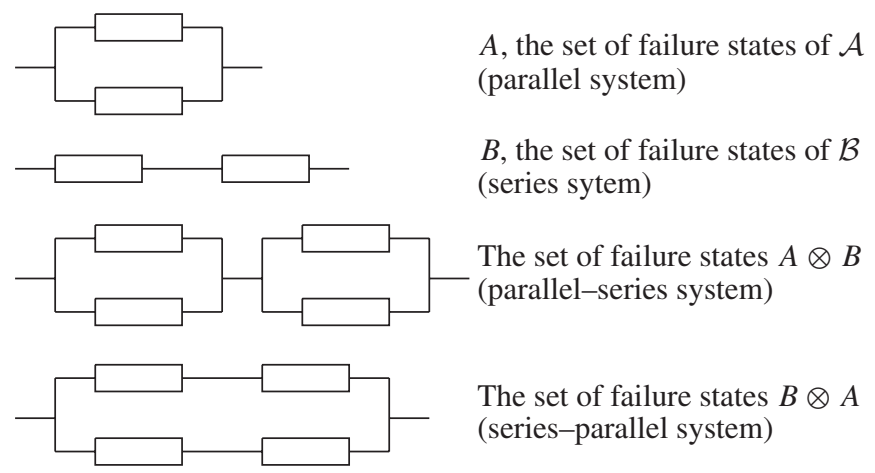

FIGURE 2: Parallel-series and series-parallel systems obtained as products.

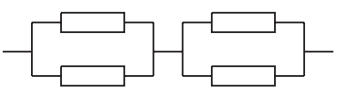

$A$, the set of failure states of $\mathcal{A}$
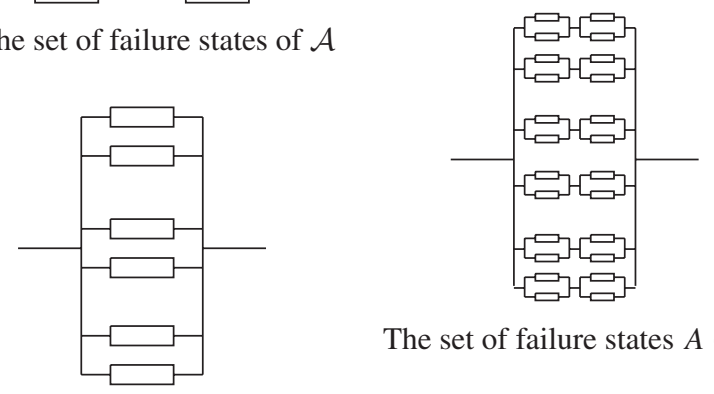

The set of failure states $A \otimes B$

$B$, the set of failure states of $\mathcal{B}$

FIgURE 3: An example of a product in reliability theory.

Proposition 4.1. Let $A \subset\{0,1\}^{r}$ and $B \subset\{0,1\}^{m}$.

1. For every $p \in[0,1]$,

$$
\mu_{m r, p}(A \otimes B)=\mu_{m, \mu_{r, p}(A)}(B) .
$$

2. If $A$ and $B$ are monotone then $A \otimes B$ is monotone.

3. If $A$ is invariant under the action of a subgroup $G$ of $\oiint_{r}$ and $B$ is invariant under the action of a subgroup $H$ of $s_{m}$, then $A \otimes B$ is invariant under the action of the subgroup $G \times H$ of the permutations of $\{1, \ldots, r\} \times\{1, \ldots, m\}$ defined by

$$
(g, h) \cdot(i, j)=(g \cdot i, h \cdot j) \quad \text { for all } i \in\{1, r\} \text { and all } j \in\{1, m\} .
$$

Proof. If $\eta_{1}, \ldots, \eta_{m}$ are independent and distributed according to the law $\mu_{r, p}$, then $\left(\mathbf{1}_{\eta_{1} \in A}, \ldots, \mathbf{1}_{\eta_{m} \in A}\right)$ has law $\mu_{m, \mu_{r, p}(A)}$. This proves the first assertion. To prove the second assertion, let $\eta$ and $\zeta$ belong to $\left(\{0,1\}^{r}\right)^{m}$ and suppose that $\eta \leq \zeta$, that is,

$$
\eta_{i} \leq \zeta_{i}, \quad \text { for all } i \in\{1, \ldots, m\} \text {. }
$$

Since $A$ is monotone,

$$
\left(\mathbf{1}_{\left\{\eta_{1} \in A\right\}}, \ldots, \mathbf{1}_{\left\{\eta_{m} \in A\right\}}\right) \preceq\left(\mathbf{1}_{\left\{\zeta_{1} \in A\right\}}, \ldots, \mathbf{1}_{\left\{\zeta_{m} \in A\right\}}\right) .
$$


Suppose now that $\eta \in A \otimes B$. Then

$$
\left(\mathbf{1}_{\left\{\eta_{1} \in A\right\}}, \ldots, \mathbf{1}_{\left\{\eta_{m} \in A\right\}}\right) \in B .
$$

Since $B$ is monotone, it follows from (4.1) and (4.2) that $\zeta \in B$, which proves the monotonicity of $A \otimes B$.

To prove the final assertion of the proposition, let $\eta \in A \otimes B$ and $(g, h) \in G \times H$ and write $\zeta=(g, h) \cdot \eta$, that is,

$$
\zeta_{i, j}=\eta_{(g, h) \cdot(i, j)}=\eta_{g \cdot i, h \cdot j}
$$

which can be restated as

$$
\zeta=\left(g \cdot \eta_{h \cdot 1}, \ldots, g \cdot \eta_{h \cdot m}\right)
$$

On the other hand,

$$
\eta=\left(\eta_{1}, \ldots, \eta_{m}\right)
$$

with $\eta_{i} \in\{0,1\}^{r}$, and

$$
\left(\mathbf{1}_{\left\{\eta_{1} \in A\right\}}, \ldots, \mathbf{1}_{\left\{\eta_{m} \in A\right\}}\right) \in B .
$$

Therefore,

$$
\begin{array}{r}
\left(\mathbf{1}_{\left\{g\left(\eta_{1}\right) \in A\right\}}, \ldots, \mathbf{1}_{\left\{g\left(\eta_{m}\right) \in A\right\}}\right) \in B, \\
h \cdot\left(\mathbf{1}_{\left\{g\left(\eta_{1}\right) \in A\right\}}, \ldots, \mathbf{1}_{\left\{g\left(\eta_{m}\right) \in A\right\}}\right) \in B,
\end{array}
$$

implying that

$$
\left(\mathbf{1}_{\left\{g\left(\eta_{h \cdot 1}\right) \in A\right\}}, \ldots, \mathbf{1}_{\left\{g\left(\eta_{h \cdot m}\right) \in A\right\}}\right) \in B .
$$

Thus, $\zeta \in A \otimes B$ and the proof is complete.

Intuitively, the first assertion in Proposition 4.1 suggests that if the threshold of $B$ is located away from both 0 and 1, then the threshold effects of $A$ and $B$ will conjugate and give rise to a threshold width the order of which will be the product of the widths of $A$ and $B$. This is indeed the case, and this is roughly the statement of Proposition 4.2. Actually, this result is valid as long as the threshold of $B$ is located away from both 0 and 1 and some additional hypotheses of homogeneity hold for the threshold widths of $A$ and $B$. When a threshold phenomenon occurs for a property $A$, it is usually true that the threshold width is homogeneous, in the sense that all the transition intervals shrink at the same speed. This allows us to consider the exact order of the threshold width, since this does not depend on the level $\varepsilon$. We will use the following definitions of homogeneity and strong homogeneity.

Definition 4.2. Let $A \subset\{0,1\}^{\alpha_{n}}$ be a nontrivial, monotone property, and let $\left(a_{n}\right)_{n \in \mathbb{N}}$ be a sequence of positive real numbers. The threshold width of the property $A$ is homogeneous of order $a_{n}$ if, for all $\beta, \gamma \in(0,1)$ such that $\beta<\gamma$,

$$
p_{A, \gamma}-p_{A, \beta}=\Theta\left(a_{n}\right) .
$$

The threshold width of the property $A$ is strongly homogeneous of order $a_{n}$ if, in addition, for all sequences of real numbers $\left(\beta_{n}\right)_{n \in \mathbb{N}}$ and $\left(\gamma_{n}\right)_{n \in \mathbb{N}}$ such that there exists an $\varepsilon \in(0,1)$ for which

$$
\varepsilon<\beta_{n}<\gamma_{n}<1-\varepsilon \text { for all } n \in \mathbb{N},
$$

we have

$$
p_{A, \gamma_{n}}-p_{A, \beta_{n}}=O\left(\left(\gamma_{n}-\beta_{n}\right) a_{n}\right)
$$


We are now able to state the main result about the width of a product.

Proposition 4.2. Let $\left(r_{n}\right)_{n \in \mathbb{N}}$ and $\left(m_{n}\right)_{n \in \mathbb{N}}$ be two nondecreasing sequences of integers, and let $A \subset\{0,1\}^{r_{n}}$ and $B \subset\{0,1\}^{m_{n}}$ be two monotone properties. Suppose that the threshold width of $A$ is strongly homogeneous of order $a_{n}$ and that the threshold width of $B$ is homogeneous of order $b_{n}$. Suppose, in addition, that the threshold of $B$ is located away from both 0 and 1 , that is, for all $\varepsilon \in(0,1)$ there exists a $\delta \in(0,1)$ such that

$$
\delta<p_{B, \varepsilon}<1-\delta \text { for all } n \in \mathbb{N} .
$$

Then the threshold of $A \otimes B \subset\{0,1\}^{r_{n} m_{n}}$ has a homogeneous width of order $a_{n} b_{n}$. Moreover, if the threshold of $A$ is located at $\alpha \in[0,1]$, then so is the threshold of $A \otimes B$.

Proof. Let $\varepsilon$ be a real number in $\left(0, \frac{1}{2}\right)$. According to Proposition 4.1,

$$
\mu_{m_{n} r_{n}, p}(A \otimes B)=\mu_{m_{n}, \mu_{r_{n}, p}(A)}(B) .
$$

Therefore, $\mu_{r_{n}, p_{A \otimes B, \varepsilon}}(A)=p_{B, \varepsilon}$ and, so,

$$
\begin{aligned}
p_{A \otimes B, \varepsilon} & =p_{A, p_{B, \varepsilon}} \\
p_{A \otimes B, 1-\varepsilon}-p_{A \otimes B, \varepsilon} & =p_{A, p_{B, 1-\varepsilon}}-p_{A, p_{B, \varepsilon}} .
\end{aligned}
$$

Since the threshold width of $B$ is of order $b_{n}$, we have

$$
p_{B, 1-\varepsilon}-p_{B, \varepsilon}=\Theta\left(b_{n}\right) .
$$

Recall that, by hypothesis, there exists a $\delta \in(0,1)$ such that

$$
\delta<p_{B, \varepsilon}<p_{B, 1-\varepsilon}<1-\delta \text { for all } n \in \mathbb{N} .
$$

Thus, the fact that $A$ has a strongly homogeneous threshold width of order $a_{n}$ (see Definition 4.2) implies that

$$
p_{A, p_{B, 1-\varepsilon}}-p_{A, p_{B, \varepsilon}}=\Theta\left(\left(p_{B, 1-\varepsilon}-p_{B, \varepsilon}\right) a_{n}\right)=\Theta\left(a_{n} b_{n}\right) .
$$

Therefore, the threshold of $A \otimes B \subset\{0,1\}^{r_{n} m_{n}}$ has a homogeneous width of order $a_{n} b_{n}$.

Now suppose that $A$ is located at $\alpha \in[0,1]$, and let $\varepsilon$ be a real number in $(0,1)$. Recall that there exists a $\delta \in(0,1)$ such that

$$
\delta<p_{B, \varepsilon}<1-\delta \quad \text { for all } n \in \mathbb{N} .
$$

Since $p_{A \otimes B, \varepsilon}=p_{A, p_{B, \varepsilon}}$,

$$
p_{A, \delta}<p_{A \otimes B, \varepsilon}<p_{A, 1-\delta} \text { for all } n \in \mathbb{N} .
$$

Thus, $p_{A \otimes B, \varepsilon}$ tends to $\alpha$ as $n$ tends to $\infty$. This completes the proof.

\section{Symmetric threshold widths between $1 / \log n$ and $1 / \sqrt{n}$}

In this section we show how to derive from Proposition 4.2 a large variety of threshold widths, ranging from $1 / \log n$ to $1 / \sqrt{n}$. To this end, we need as elementary building blocks some properties the thresholds of which are easy to study, and which we shall eventually combine in order to obtain the desired threshold widths. These blocks will be taken from the reliability examples of Section 3 . 
Recall that, for any $k \in\{1, \ldots, n\}$, we denote by $A_{k, n}$ the following subset of configurations in $\{0,1\}^{n}$ (see Example 3.1):

$$
A_{k, n}=\left\{x \in\{0,1\}^{n}: \sum_{i=1}^{n} x_{i} \geq k\right\} .
$$

In what follows we shall use $A_{\lfloor n / 2\rfloor, n}$ and $A_{1, r} \otimes A_{m-1, m}$, for different values of $n, r$, and $m$.

Lemma 5.1. Let $A=\left(A_{\lfloor n / 2\rfloor, n}\right)_{n \in \mathbb{N}^{*}}$. Then, for every $n \in \mathbb{N}^{*}$,

$$
\tau\left(A_{\lfloor n / 2\rfloor, n}, \varepsilon\right) \leq 2 \sqrt{\frac{\log (1 / \varepsilon)}{2 n}} .
$$

Moreover, A has a strongly homogeneous threshold located at $\frac{1}{2}$, with a width of order $1 / \sqrt{n}$.

Proof. A simple way to show that $A$ has a threshold located at $\frac{1}{2}$ with a width of order $1 / \sqrt{n}$ is to use the concentration property of the binomial law. Indeed, Hoeffding's inequality [14] ensures that

$$
\mu_{p}\left(\sum_{k=1}^{n} x_{i}-n p>\lambda \sqrt{n}\right) \leq \mathrm{e}^{-2 \lambda^{2}} \text { for all } \lambda>0
$$

and that

$$
\mu_{p}\left(\sum_{k=1}^{n} x_{i}-n p<-\lambda \sqrt{n}\right) \leq \mathrm{e}^{-2 \lambda^{2}} \text { for all } \lambda>0 .
$$

Let $\varepsilon \in(0,1)$ and let $c=\sqrt{\log (1 / \varepsilon) / 2}$ or, equivalently, $\exp \left(-2 c^{2}\right)=\varepsilon$. If $p(\varepsilon)$ is such that $\mu_{n, p(\varepsilon)}\left(A_{\lfloor n / 2\rfloor, n}\right)=\varepsilon$, then $\lfloor n / 2\rfloor$ is at most a distance of order $\sqrt{n}$ from $n p(\varepsilon)$. Inequalities (5.1) and (5.2) imply that

$$
\lfloor n / 2\rfloor-\sqrt{\frac{n \log (1 /(1-\varepsilon))}{2}} \leq n p(\varepsilon) \leq\lfloor n / 2\rfloor+\sqrt{\frac{n \log (1 /(1-\varepsilon))}{2}} .
$$

Therefore, the threshold of $A_{\lfloor n / 2\rfloor, n}$ is located at $\frac{1}{2}$, that is,

$$
p(\varepsilon) \rightarrow \frac{1}{2} \quad \text { for all } \varepsilon \in(0,1),
$$

and its threshold width is at most of order $1 / \sqrt{n}$, that is,

$$
\tau\left(A_{\lfloor n / 2\rfloor, n}, \varepsilon\right) \leq 2 \sqrt{\frac{\log (1 /(\varepsilon))}{2 n}} \text { for all } n \in \mathbb{N}^{*} \text { and all } \varepsilon \in\left(0, \frac{1}{2}\right) .
$$

To see that this is the right order, we can express the derivative $\mathrm{d} \mu_{p}(A) / \mathrm{d} p$ as follows:

$$
\begin{aligned}
\frac{\mathrm{d} \mu_{p}(A)}{\mathrm{d} p} & =\sum_{x \in\{0,1\}^{n}} \mathbf{1}_{A}(x) \frac{\mathrm{d} \mu_{p}(x)}{\mathrm{d} p}, \\
& =\sum_{i=1}^{n} \sum_{x \in\{0,1\}^{n}} \mathbf{1}_{A}(x) \frac{x_{i}-p}{p(1-p)} \mu_{p}(x), \\
& =\frac{1}{p(1-p)} \operatorname{cov}\left(\mathbf{1}_{A}, S_{n}\right) .
\end{aligned}
$$


Then, by the Cauchy-Schwarz inequality,

$$
\begin{aligned}
\frac{\mathrm{d} \mu_{p}(A)}{\mathrm{d} p} & \leq \frac{1}{p(1-p)} \sqrt{\mu_{p}(A)\left(1-\mu_{p}(A)\right)} \sqrt{n p(1-p),} \\
\frac{\mathrm{d} \mu_{p}(A)}{\mathrm{d} p} & \leq \frac{\sqrt{\mu_{p}(A)\left(1-\mu_{p}(A)\right)}}{\sqrt{p(1-p)}} \sqrt{n}, \\
\frac{1}{\sqrt{\mu_{p}(A)\left(1-\mu_{p}(A)\right)}} \frac{\mathrm{d} \mu_{p}(A)}{\mathrm{d} p} & \leq \frac{\sqrt{n}}{\sqrt{p(1-p)}} .
\end{aligned}
$$

It is easy to integrate this differential inequation. Let us define

$$
J(x)=(1-x) \sqrt{x(1-x)}+\arctan \left(\sqrt{\frac{x}{1-x}}\right) .
$$

Then

$$
J^{\prime}(x)=\frac{1}{\sqrt{x(1-x)}} \quad \text { for all } x \in(0,1) .
$$

Therefore, integrating (5.3) between $p(\varepsilon)$ and $p(1-\varepsilon)$ gives

$$
J(1-\varepsilon)-J(\varepsilon) \leq \sqrt{n}(J(p(1-\varepsilon))-J(p(\varepsilon))) .
$$

As $n$ tends to $\infty, p(\varepsilon)$ and $p(1-\varepsilon)$ tend to $\frac{1}{2}$. Therefore,

$$
\begin{aligned}
& J(1-\varepsilon)-J(\varepsilon) \leq \sqrt{n} J^{\prime}\left(\frac{1}{2}\right)(p(1-\varepsilon)-p(\varepsilon)+o(p(1-\varepsilon)-p(\varepsilon))), \\
& p(1-\varepsilon)-p(\varepsilon) \leq \frac{J(1-\varepsilon)-J(\varepsilon)}{2 \sqrt{n}}+o\left(\frac{1}{\sqrt{n}}\right) .
\end{aligned}
$$

Therefore, $\tau(A, \varepsilon)=O(1 / \sqrt{n})$.

Finally, to prove the strong homogeneity of the width, we need a sharp minorization of $\mathrm{d} \mu_{p}(A) / \mathrm{d} p$. A smooth way to do this is to use one of the discrete isoperimetric inequalities of Margulis and Talagrand. The work of Margulis [21] has motivated a number of increasingly accurate discrete isoperimetric inequalities [26], [2], [3], [27]. For example, let $\phi$ denote the Gaussian density, that is, $\phi(t)=(1 / \sqrt{2 \pi}) \mathrm{e}^{-t^{2} / 2}$, and $\Phi$ the Gaussian cumulative distribution, that is, $\Phi(x)=\int_{-\infty}^{x} \phi(t) \mathrm{d} t$. From the main result of [27] it follows that

$$
\frac{\mathrm{d} \mu_{p}(A)}{\mathrm{d} p} \geq \frac{\sqrt{n}}{p \sqrt{\log (1 / p)}} \Psi\left(\mu_{p}(A)\right),
$$

where $\Psi$ stands for $\phi \circ \Phi^{-1}$. Therefore,

$$
\frac{\mathrm{d} \mu_{p}(A)}{\mathrm{d} p} \geq \sqrt{n \mathrm{e}} \Psi\left(\mu_{p}(A)\right) .
$$

Let $\left(\beta_{n}\right)_{n \in \mathbb{N}}$ and $\left(\gamma_{n}\right)_{n \in \mathbb{N}}$ be two sequences of real numbers in $(0,1)$. Integrating this inequality between $p\left(\beta_{n}\right)$ and $p\left(\gamma_{n}\right)$ leads to

$$
p\left(\gamma_{n}\right)-p\left(\beta_{n}\right) \leq \frac{1}{\sqrt{n \mathrm{e}}} \int_{\beta_{n}}^{\gamma_{n}} \Psi(u) \mathrm{d} u .
$$


Now suppose that there exists an $\varepsilon \in(0,1)$ such that

$$
\varepsilon<\beta_{n}<\gamma_{n}<1-\varepsilon \text { for all } n \in \mathbb{N} .
$$

Since $\Psi$ is continuous and strictly positive on $(0,1)$, we have

$$
\int_{\beta_{n}}^{\gamma_{n}} \Psi(u) \mathrm{d} u=\Theta\left(\gamma_{n}-\beta_{n}\right)
$$

Finally,

$$
p\left(\gamma_{n}\right)-p\left(\beta_{n}\right)=O\left(\left(\gamma_{n}-\beta_{n}\right) / \sqrt{n}\right)
$$

and, thus, the strong homogeneity of the threshold width of $A$ holds.

Now consider a parallel-series system composed of $r$ blocks containing $m$ components (see Example 3.2). The system fails as soon as a block fails, and a block fails if all of its components fail. The failure subset of such a system is $A_{m, m} \otimes A_{1, r}$. It is symmetric and monotone. Its probability can easily be explicitly derived:

$$
\mu_{p}\left(A_{m, m} \otimes A_{1, r}\right)=1-\left(1-p^{m}\right)^{r} .
$$

Thus, for any $\alpha \in(0,1)$,

$$
p_{\alpha}=\left(1-(1-\alpha)^{1 / r}\right)^{1 / m} .
$$

Lemma 5.2. For every $k \in \mathbb{N}^{*}$, let $B_{k}$ be the following parallel-series failure subset:

$$
B_{k}=A_{\left\lfloor\log _{2} k\right\rfloor,\left\lfloor\log _{2} k\right\rfloor} \otimes A_{1,\left\lfloor k / \log _{2} k\right\rfloor} \subset\{0,1\}^{K},
$$

where $K=\left\lfloor\log _{2} k\right\rfloor\left\lfloor k / \log _{2} k\right\rfloor$. The property $B_{k}$ has a sharp threshold located at $\frac{1}{2}$ with homogeneous width of order $1 / \log K$. More precisely, the threshold width of $B_{k}$ has the following asymptotic expansion:

$$
\tau\left(B_{k}, \varepsilon\right)=\frac{1}{2 \log K} \log \frac{\log (1 / \varepsilon)}{\log (1 /(1-\varepsilon))}+o\left(\frac{1}{\log _{2} K}\right) .
$$

Proof. Let $\alpha \in(0,1)$. We have

$$
\begin{aligned}
p_{\alpha} & =\left(1-(1-\alpha)^{1 /\left\lfloor k / \log _{2} k\right\rfloor}\right)^{1 /\left\lfloor\log _{2} k\right\rfloor} \\
& =\left(\frac{\log _{2} k}{k} \log \frac{1}{1-\varepsilon}+o\left(\frac{\log _{2} k}{k}\right)\right)^{1 / \log _{2} k+o(1 / \log k)} \\
& =\exp \left(-\frac{\log k}{\log _{2} k}+\frac{\log \log _{2} k}{\log _{2} k}+\frac{\log \log (1 /(1-\varepsilon))}{\log _{2} k}+o\left(\frac{1}{\log k}\right)\right) \\
& =\frac{1}{2}\left(1+\frac{\log \log _{2} k}{\log _{2} k}+\frac{\log \log (1 /(1-\varepsilon))}{\log _{2} k}+o\left(\frac{1}{\log k}\right)\right) .
\end{aligned}
$$

Therefore, the threshold of $B_{k}$ is located at $\frac{1}{2}$. Moreover, for any $\varepsilon \in\left(0, \frac{1}{2}\right)$,

$$
\tau\left(B_{k}, \varepsilon\right)=\frac{1}{2 \log k} \log 2 \log \frac{\log (1 / \varepsilon)}{\log (1 /(1-\varepsilon))}+o\left(\frac{1}{\log k}\right) .
$$

Since $K(k)=\left\lfloor\log _{2} k\right\rfloor \times\left\lfloor k / \log _{2} k\right\rfloor$ is equivalent to $k$ as $k$ tends to $\infty$,

$$
\tau\left(B_{k}, \varepsilon\right)=\frac{1}{2 \log K} \log 2 \log \frac{\log (1 / \varepsilon)}{\log (1 /(1-\varepsilon))}+o\left(\frac{1}{\log K}\right) .
$$

Thus, $B_{k}$ has a threshold with homogeneous width of order $1 / \log K$. 
We are now able to prove our main result.

Theorem 5.1. Let c $(n)$ be a nondecreasing sequence of integers such that

$$
\log n \leq c(n) \leq \sqrt{n} \text { for all } n \in \mathbb{N}^{*}
$$

and suppose that

$$
c(2 n)=\Theta(c(n)) .
$$

Then there exist an increasing sequence of integers $(N(n))_{n \in \mathbb{N}^{*}}$ and a monotone, symmetric property $C \subset\{0,1\}^{N(n)}$ whose threshold is located at $\frac{1}{2}$ and has width of order $1 / c(N(n))$.

Remark 5.1. The condition $c(2 n)=\Theta(c(n))$ implies that $c$ increases rather smoothly. Because of the way we chose to build the property mentioned in Theorem 5.1, we cannot avoid this condition. Of course, any condition of the type $c(r n)=\Theta(c(n))$ with $r$ an integer strictly greater than 1 would be sufficient, since $c$ is nondecreasing. Note that this condition is satisfied for most natural choices of nondecreasing function. Nevertheless, it is possible to imagine 'unnatural' examples in which this condition is not satisfied. Indeed, let $\left(a_{j}\right)_{j \in \mathbb{N}^{*}}$ be the increasing sequence of integers recursively defined by

$$
a_{0}=2, \quad a_{j+1}=\mathrm{e}^{\left\lfloor\sqrt{2 a_{j}}\right\rfloor}, \quad j \geq 0 .
$$

Note that $a_{j+1}-1 \geq 2 a_{j}$ for every $j \geq 1$. Let $c(n)$ be the nondecreasing sequence of integers defined by

$$
c(n)= \begin{cases}\log (n) & \text { for all } n \in\left[a_{j}, 2 a_{j}-1\right], \\ \sqrt{2 a_{j}} & \text { for all } n \in\left[2 a_{j}, a_{j+1}-1\right] .\end{cases}
$$

On one hand

$$
\log (n) \leq c(n) \leq \sqrt{n} \text { for all } n \in \mathbb{N}^{*}
$$

and on the other hand

$$
c\left(a_{j}\right)=\log a_{j} \quad \text { and } \quad c\left(2 a_{j}\right)=\sqrt{2 a_{j}} \quad \text { for all } j \in \mathbb{N}^{*} .
$$

Therefore, $c(2 n) / c(n)$ is not bound from above.

Proof of Theorem 5.1. For any integer $k \geq 2$, let $B_{k}$ be the failure subset of a parallel-series system composed of $\left\lfloor k / \log _{2} k\right\rfloor$ blocks each containing $\left\lfloor\log _{2} k\right\rfloor$ components. Suppose that $1 \leq a(n) \leq n$. Let us define the integer

$$
N=\left\lfloor\frac{n}{a(n)}\right\rfloor a(n)
$$

and the following monotone, symmetric subset of $\{0,1\}^{N}$ :

$$
C_{N}=A_{\lfloor a(n) / 2\rfloor, a(n)} \otimes B_{\lfloor n / a(n)\rfloor} .
$$

According to Lemma 5.1, $A_{\lfloor a(n) / 2\rfloor, a(n)}$ has a threshold located at $\frac{1}{2}$ with strongly homogeneous width of order $1 / \sqrt{a(n)}$. From Lemma 5.2, $B_{\lfloor n / a(n)\rfloor}$ has a threshold located at $\frac{1}{2}$ with homogeneous width of order $1 / \log \lfloor n / a(n)\rfloor$. Therefore, we can deduce from Proposition 4.2 that $C_{N}$ has a threshold located at $\frac{1}{2}$ with homogeneous width of order $\sqrt{a(n)} \log \lfloor n / a(n)\rfloor$. 
It is apparent that this device allows us to build properties with threshold widths of nearly any order between $1 / \log N$ and $1 / \sqrt{N}$.

Indeed, let $\phi_{n}$ be the following function:

$$
\phi_{n}:[1, n] \rightarrow \mathbb{R}, \quad x \mapsto\left(\log \frac{n}{x}\right)^{2} .
$$

The derivative of $\phi_{n}$ is easy to compute:

$$
\phi_{n}^{\prime}(x)=\log \frac{n}{x} \log \frac{n \mathrm{e}^{-2}}{x} \quad \text { for all } x \in[1, n] .
$$

Therefore, $\phi_{n}$ is a bijection from $\left[1, n / \mathrm{e}^{2}\right]$ to $\left[(\log n)^{2}, 4 n / \mathrm{e}^{2}\right]$. Let $c(n)$ be a sequence of integers such that

$$
\log n \leq c(n) \leq \sqrt{n} \text { for all } n \in \mathbb{N}^{*} .
$$

Define $\tilde{c}(n)=\inf \{c(n), 2 \sqrt{n} / \mathrm{e}\}$. Thus,

$$
(\log n)^{2} \leq \tilde{c}(n)^{2} \leq 4 n / \mathrm{e}^{2} \text { for all } n \in \mathbb{N}^{*} .
$$

Let $a(n)=\phi_{n}^{-1}\left(\tilde{c}(n)^{2}\right) \in\left[1, n / \mathrm{e}^{2}\right]$. The subset $C_{N}$ has a threshold width of order $1 / \sqrt{\phi_{n}(a(n))}=1 / \tilde{c}(n)$. It is clear that $\tilde{c}(n)$ and $c(n)$ are of the same order as $n$ tends to $\infty$. Therefore, $C_{N}$ has a threshold width of order $1 / c(n)$. Furthermore, suppose that $c$ is nondecreasing. Since $n \leq N(n) \leq 2 n$, we have

$$
c(n) \leq c(N(n)) \leq c(2 n) \text { for all } n \in \mathbb{N}^{*} .
$$

Now suppose that $c(2 n)=\Theta(c(n))$. Then $c(N(n))=\Theta(c(n))$. Finally, $C_{N}$ has a threshold width of order $1 / c(N(n))$. The result follows.

\section{How to obtain a sharp threshold from two coarse ones}

When the localisation of the threshold of $B$ is not bounded away from 0 and 1, Proposition 4.2 is useless in describing the threshold width of $A \otimes B$. Moreover, its conclusion is no longer valid. Indeed, consider the failure subset,

$$
B_{k}=A_{\left\lfloor\log _{2} k\right\rfloor,\left\lfloor\log _{2} k\right\rfloor} \otimes A_{1,\left\lfloor k / \log _{2} k\right\rfloor} \subset\{0,1\}^{K},
$$

defined in Lemma 5.2. We can easily compute the probability of $A_{1, n}$ to be

$$
\mu_{p}\left(A_{1, n}\right)=1-(1-p)^{n}
$$

therefore,

$$
p(\varepsilon)=1-(1-\varepsilon)^{1 / n} .
$$

Thus, as $n$ tends to $\infty, p(\varepsilon)$ is equivalent to $\log (1 /(1-\varepsilon)) / n$ and $\tau\left(A_{1, n}, \varepsilon\right)$ is equivalent to $\log ((1-\varepsilon) / \varepsilon) / n$. This is a typical example of a coarse threshold. We similarly obtain

$$
\mu_{p}\left(A_{n-1, n}\right)=p^{n}
$$

whence

$$
p(\varepsilon)=\varepsilon^{1 / n} .
$$


Thus, as $n$ tends to $\infty, p(\varepsilon)$ is equivalent to $1-\log (1 / \varepsilon) / n$ and $\tau\left(A_{n-1, n}, \varepsilon\right)$ is again equivalent to $\log ((1-\varepsilon) / \varepsilon) / n$.

Consequently, $A_{\left\lfloor\log _{2} k\right\rfloor,\left\lfloor\log _{2} k\right\rfloor}$ and $A_{1,\left\lfloor k / \log _{2} k\right\rfloor}$ have threshold widths of respective orders $1 / \log k$ and $\log k / k$. According to Lemma 5.2, their product is a subset of $\{0,1\}^{K}$, where $K=\left\lfloor\log _{2} k\right\rfloor \times\left\lfloor k / \log _{2} k\right\rfloor$, and has a threshold width of order $1 / \log K$. This is much bigger than $1 / K$, the order we would find were the conclusion of Proposition 4.2 to remain valid.

Nevertheless, in this example we witness an interesting phenomenon. The two subsets $A_{\left\lfloor\log _{2} k\right\rfloor,\left\lfloor\log _{2} k\right\rfloor}$ and $A_{1,\left\lfloor k / \log _{2} k\right\rfloor}$ of $\{0,1\}^{K}$ clearly have coarse thresholds, but their product has a sharp one. We shall prove this to be very general behaviour: if $A$ and $B$ have thresholds located respectively at 0 and 1 , then, even if these are coarse, their product $A \otimes B$ has a sharp threshold. To prove this we shall use a well-known tensorisation property of the entropy. The major role of this property in concentration and threshold topics has been pointed out many times (see, for example, [20], [6], and [25]). First, let us recall the definition of the entropy of a nonnegative function $f$ on a probability space $(\mathcal{X}, \mu)$ :

$$
\text { Ent }_{\mu}(f)=\int f(x) \log f(x) \mathrm{d} \mu(x)-\int f(x) \mathrm{d} \mu(x) \log \int f(x) \mathrm{d} \mu(x) .
$$

Entropy satisfies the following tensorisation inequality (see, for example, [20, Proposition 5.6]): for every nonnegative function $f$ on $\{0,1\}^{n}$,

$$
\sum_{i=1}^{n} \mathrm{E}_{\mu_{p}}\left(\operatorname{Ent}_{x_{i}}(f)\right) \geq \operatorname{Ent}_{\mu_{p}}(f)
$$

where in Ent $\mu_{i}$ only the $i$ th coordinate of $\mu$ is involved in the integration. The following lemma is the key towards the main result of this section, Proposition 6.1.

Lemma 6.1. Let $A$ be a monotone subset of $\{0,1\}^{n}$. Then, for every $p \in[0,1]$,

$$
\begin{aligned}
p \log \frac{1}{p} \frac{\mathrm{d} \mu_{p}(A)}{\mathrm{d} p} & \geq \mu_{p}(A) \log \frac{1}{\mu_{p}(A)}, \\
(1-p) \log \frac{1}{1-p} \frac{\mathrm{d} \mu_{p}(A)}{\mathrm{d} p} & \geq\left(1-\mu_{p}(A)\right) \log \frac{1}{1-\mu_{p}(A)} .
\end{aligned}
$$

Proof. The following formula is easily obtained by considering the derivative of $\mu_{p}(x)$ with respect to $p$. For any real function $f$ on $\{0,1\}^{n}$,

$$
\frac{\mathrm{d}}{\mathrm{d} p} \int f(x) \mathrm{d} \mu_{p}(x)=\sum_{i=1}^{n} \int \nabla_{i} f(x) \mathrm{d} \mu_{p}(x),
$$

where, for all $x \in\{0,1\}^{n}$,

$$
\nabla_{i} f(x)=f\left(x_{1}, \ldots, x_{i-1}, 1, x_{i+1}, \ldots, x_{n}\right)-f\left(x_{1}, \ldots, x_{i-1}, 0, x_{i+1}, \ldots, x_{n}\right) .
$$

On the other hand, if $A$ is a monotone subset then

$$
\text { Ent }_{x_{i}}\left(\mathbf{1}_{A}\right)=\int \mathbf{1}_{A} \log \frac{\mathbf{1}_{A}}{\int \mathbf{1}_{A} \mathrm{~d} x_{i}} \mathrm{~d} x_{i}=p \log \frac{1}{p} \nabla_{i} \mathbf{1}_{A} .
$$


Therefore,

$$
\sum_{i=1}^{n} \mathrm{E}_{\mu_{p}}\left(\operatorname{Ent}_{x_{i}}(f)\right)=p \log \frac{1}{p} \frac{\mathrm{d} \mu_{p}(A)}{\mathrm{d} p} .
$$

Note that, for any subset $A$,

$$
\operatorname{Ent}_{\mu_{p}}\left(\mathbf{1}_{A}\right)=\mu_{p}(A) \log \frac{1}{\mu_{p}(A)} .
$$

Thus, when applied to $f=\mathbf{1}_{A},(6.1)$ gives

$$
p \log \frac{1}{p} \frac{\mathrm{d} \mu_{p}(A)}{\mathrm{d} p} \geq \mu_{p}(A) \log \frac{1}{\mu_{p}(A)} .
$$

Now note that

$$
\operatorname{Ent}_{x_{i}}\left(\mathbf{1}_{A^{\mathrm{c}}}\right)=\int \mathbf{1}_{A^{\mathrm{c}}} \log \frac{\mathbf{1}_{A^{\mathrm{c}}}}{\int \mathbf{1}_{A^{\mathrm{c}}} \mathrm{d} x_{i}} \mathrm{~d} x_{i}=p \log \frac{1}{p} \nabla_{i} \mathbf{1}_{A} .
$$

Since

$$
\operatorname{Ent}_{\mu_{p}}\left(\mathbf{1}_{A^{\mathrm{c}}}\right)=\mu_{p}\left(A^{\mathrm{c}}\right) \log \frac{1}{\mu_{p}\left(A^{\mathrm{c}}\right)},
$$

when applied to $f=\mathbf{1}_{A^{\mathrm{c}}}(6.1)$ gives

$$
(1-p) \log \frac{1}{1-p} \frac{\mathrm{d} \mu_{p}(A)}{\mathrm{d} p} \geq\left(1-\mu_{p}(A)\right) \log \frac{1}{1-\mu_{p}(A)} .
$$

Proposition 6.1. Let $\left(r_{n}\right)_{n \in \mathbb{N}}$ and $\left(m_{n}\right)_{n \in \mathbb{N}}$ be two increasing sequences of integers, and let $A \subset\{0,1\}^{r_{n}}$ and $B \subset\{0,1\}^{m_{n}}$ be two nontrivial, monotone properties. Suppose that the threshold of $A$ is located at 1 and that the threshold of $B$ is located at 0 , that is, for all $\varepsilon \in(0,1)$,

$$
p_{A, \varepsilon} \rightarrow 1 \text { as } n \rightarrow \infty
$$

and

$$
p_{B, \varepsilon} \rightarrow 0 \text { as } n \rightarrow \infty \text {. }
$$

Then the thresholds of $A \otimes B$ and $B \otimes A$ are sharp.

Proof. First, let us remark that $A \otimes B$ has a coarse threshold if and only if, for every $\varepsilon \in(0,1)$,

$$
\left.p_{A \otimes B, \varepsilon}\left(1-p_{A \otimes B, \varepsilon}\right) \frac{\mathrm{d} \mu_{p}(A \otimes B)}{\mathrm{d} p}\right|_{p=p_{A \otimes B, \varepsilon}} \rightarrow \infty \quad \text { as } n \rightarrow \infty
$$

(see [12] or [24, p. 99]). Now, for all monotone subsets $A$ and $B$ and every $p \in(0,1)$, from Proposition 4.1 we have

$$
\mu_{p}(A \otimes B)=\mu_{\mu_{p}(A)}(B) .
$$

Let us denote by $f(p)$ the quantity $\mu_{p}(A \otimes B)$. We therefore have

$$
f^{\prime}(p)=\left.\frac{\mathrm{d} \mu_{p}(A)}{\mathrm{d} p} \frac{\mathrm{d} \mu_{q}(B)}{\mathrm{d} q}\right|_{q=\mu_{p}(A)} .
$$


According to Lemma 6.1,

$$
\begin{aligned}
\frac{\mathrm{d} \mu_{p}(A)}{\mathrm{d} p} & \geq \frac{\mu_{p}(A) \log \left(1 / \mu_{p}(A)\right)}{p \log (1 / p)} \\
\left.\frac{\mathrm{d} \mu_{q}(B)}{\mathrm{d} q}\right|_{q=\mu_{p}(A)} & \geq \frac{(1-f(p)) \log (1 /(1-f(p)))}{\left(1-\mu_{p}(A)\right) \log \left(1 /\left(1-\mu_{p}(A)\right)\right)} .
\end{aligned}
$$

Thus,

$$
f^{\prime}(p) \geq \frac{(1-f(p)) \log (1 /(1-f(p)))}{p \log (1 / p)} \frac{\mu_{p}(A) \log \left(1 / \mu_{p}(A)\right)}{\left(1-\mu_{p}(A)\right) \log \left(1 /\left(1-\mu_{p}(A)\right)\right)} .
$$

We shall focus on $A \otimes B$, since the threshold of $B \otimes A$ can be treated in the same way by switching the roles of $A$ and $B$. Suppose that the threshold of $B$ is located at 0 , and let $\varepsilon \in(0,1)$. From $(6.3)$, we obtain

$$
p_{A \otimes B, \varepsilon}=p_{A, p_{B, \varepsilon}} .
$$

Let us define $p_{\varepsilon}:=p_{A \otimes B, \varepsilon}$. From (6.4) we obtain

$$
f^{\prime}\left(p_{\varepsilon}\right) \geq \frac{(1-\varepsilon) \log (1 /(1-\varepsilon))}{p_{\varepsilon} \log \left(1 / p_{\varepsilon}\right)} \frac{p_{B, \varepsilon} \log \left(1 / p_{B, \varepsilon}\right)}{\left(1-p_{B, \varepsilon}\right) \log \left(1 /\left(1-p_{B, \varepsilon}\right)\right)} .
$$

Since $p_{B, \varepsilon}$ tends to 0 as $n$ tends to $\infty$, we have

$$
\begin{aligned}
f^{\prime}\left(p_{\varepsilon}\right) & \geq \frac{(1-\varepsilon) \log (1 /(1-\varepsilon))}{p_{\varepsilon} \log \left(1 / p_{\varepsilon}\right)}\left(\log \frac{1}{p_{B, \varepsilon}}+o(1)\right), \\
p_{\varepsilon}\left(1-p_{\varepsilon}\right) f^{\prime}\left(p_{\varepsilon}\right) & \geq(1-\varepsilon) \log \frac{1}{1-\varepsilon} \frac{\left(1-p_{\varepsilon}\right)}{\log \left(1 / p_{\varepsilon}\right)}\left(\log \frac{1}{p_{B, \varepsilon}}+o(1)\right) .
\end{aligned}
$$

Since $\log (1 / x)$ is equivalent to $1-x$ as $x$ tends to 1 , if $p_{\varepsilon}$ is bounded away from 0 then inequality (6.5) implies that $A \otimes B$ has a sharp threshold. If $p_{\varepsilon}$ is not bounded away from 0 then we need to show that having the location of $A$ at 1 implies that $\log \left(1 / p_{\varepsilon}\right)$ is asymptotically negligible compared to $\log \left(1 / p_{B, \varepsilon}\right)$. This follows from (6.2). Indeed, integrating this inequality between $p_{\varepsilon}=p_{A, p_{B, \varepsilon}}$ and $p_{\varepsilon}=p_{A, \varepsilon}$ yields

$$
\begin{aligned}
{\left[\log \frac{1}{\left|\log \mu_{p}(A)\right|}\right]_{p_{A, p_{B, \varepsilon}}}^{p_{A, \varepsilon}} } & \geq\left[\log \frac{1}{|\log p|}\right]_{p_{A, p_{B, \varepsilon}}}^{p_{A, \varepsilon}}, \\
\log \frac{1}{|\log \varepsilon|}-\log \frac{1}{\left|\log p_{B, \varepsilon}\right|} & \geq \log \frac{1}{\left|\log p_{A, \varepsilon}\right|}-\log \frac{1}{\mid \log p_{A, p_{B, \varepsilon} \mid}}, \\
\log \frac{\log \left(1 / p_{A, p_{B, \varepsilon}}\right)}{\log \left(1 / p_{B, \varepsilon}\right)} & \leq \log \frac{1}{\log (1 / \varepsilon)}+\log \frac{1}{\left|\log p_{A, \varepsilon}\right|}, \\
\frac{\log \left(1 / p_{A, p_{B, \varepsilon}}\right)}{\log \left(1 / p_{B, \varepsilon}\right)} & \leq \frac{\log \left(1 / p_{A, \varepsilon}\right)}{\log (1 / \varepsilon)} .
\end{aligned}
$$

Since $p_{A, \varepsilon}$ tends to 1 as $n$ tends to $\infty, \log \left(1 / p_{A \otimes B, \varepsilon}\right)$ is asymptotically negligible in comparison with $\log \left(1 / p_{B, \varepsilon}\right)$. 


\section{Acknowledgements}

I would like to thank Bernard Ycart for introducing me to threshold phenomena and for his patient guidance throughout my dealing with this topic. I am grateful to an anonymous referee for some helpful comments.

\section{References}

[1] Barlow, R. and Proschan, F. (1965). Mathematical Theory of Reliability. John Wiley, New York.

[2] Bовкоv, S. (1997). An isoperimetric inequality on the discrete cube, and an elementary proof of the isoperimetric inequality in Gauss space. Ann. Prob. 25, 206-214.

[3] Bobkov, S. And Götze, F. (1999). Discrete isoperimetric and Poincaré-type inequalities. Prob. Theory Relat. Fields 114, 245-277.

[4] Bollobás, B. (1985). Random Graphs. Academic Press, London.

[5] Bollobás, B. et al. (2001). The scaling window of the 2-SAT transition. Random Structures Algorithms 18, 201-256.

[6] Boucheron, S., Lugosi, G. and Massart, P. (2003). Concentration inequalities using the entropy method. Ann. Prob. 31, 1583-1614.

[7] Bourgain, J. et al. (1992). The influence of variables in product spaces. Israel J. Math. 77, 55-64.

[8] Compton, K. (1989). 0-1 laws in logic and combinatorics. In Algorithms and Order, ed. I. Rival, Kluwer, Dordrecht, pp. 353-383.

[9] Coupier, D., Desolneux, A. And Ycart, B. (2005). Image denoising by statistical area thresholding. J. Math. Imag. Vision 22, 183-197.

[10] Creignou, N. and Daudé, H. (1999). Satisfiability threshold for random XOR-CNF formulas. Discrete Appl. Math. 96/97, 41-53.

[11] Friedgut, E. (1999). Sharp threshold of graph properties and the $k$-sat problem. J. Amer. Math. Soc. 12, 10171054.

[12] Friedgut, E. and Kalai, G. (1996). Every monotone graph property has a sharp threshold. Proc. Amer. Math. Soc. 124, 2993-3002.

[13] Grimmett, G. (1989). Percolation. Springer, New York.

[14] Hoeffoing, W. (1963). Probability inequalities for sums of bounded random variables. J. Amer. Statist. Assoc. $\mathbf{5 8}, 13-30$.

[15] KoŁowrocki, K. (1994). Limit reliability functions of some series-parallel and parallel-series systems. Appl. Math. Comput. 62, 129-151.

[16] KoŁowrocki, K. (1994). On limiting forms of the reliability functions sequence of the parallel-series system. Appl. Math. Comput. Sci. 4, 575-590.

[17] KoŁowrocki, K. (2001). Asymptotic approach to reliability evaluation of a rope transportation system. Reliab. Eng. System Safety 71, 57-64.

[18] KoŁowrocki, K. (2004). Reliability of Large Systems. Elsevier, Amsterdam.

[19] Kontoleon, J. (1980). Reliability determination of a $r$-successive-out-of- $n$ : $f$ system. IEEE Trans. Reliab. 29, 437.

[20] Ledoux, M. (1996). On Talagrand's deviation inequalities for product measures. ESAIM Prob. Statist. 1, 63-87.

[21] Margulis, G. (1974). Probabilistic characteristics of graphs with large connectivity. Prob. Pedarachi Inf. 10, 101-108 (in Russian).

[22] Paroissin, C. And YCART, B. (2003). Zero-one law for the non-availability of multistate repairable systems. Internat. J. Reliab. 10, 311-322.

[23] Paroissin, C. ANd Ycart, B. (2004). Central limit theorem for hitting times of functionals of Markov jump processes. ESAIM Prob. Statist. 8, 66-75.

[24] Rossignol, R. (2005). Largeur de seuil dans les lois du zéro-un. Doctoral Thesis, Université Paris 5. Available at http://www.math-info.univ-paris5.fr/ rost/these_pdf.pdf.

[25] Rossignol, R. (2006). Threshold for monotone symmetric properties through a logarithmic Sobolev inequality. Ann. Prob. 34, 1707-1725.

[26] Talagrand, M. (1993). Isoperimetry, logarithmic Sobolev inequalities on the discrete cube, and Margulis' graph connectivity theorem. Geom. Funct. Anal. 3, 295-314.

[27] Tillich, J.-P. AND ZÉMOR, G. (2000). Discrete isoperimetric inequalities and the probability of a decoding error. Combin. Prob. Comput. 9, 465-479. 